\title{
Dynamics of a Bertrand duopoly with differentiated products and nonlinear costs: analysis, comparisons and new evidences
}

\author{
Serena Brianzoni ${ }^{a *}$ Luca Gori ${ }^{b}$, Elisabetta Michetti ${ }^{c}$
}

\begin{abstract}
This paper studies mathematical properties and dynamics of a duopoly with price competition and horizontal product differentiation by introducing quadratic production costs (decreasing returns to scale), thus extending the model with linear costs (constant returns to scale) of Fanti et al. (2013). The economy is described by a two-dimensional non-invertible discrete time dynamic system. The paper first determines fixed points and other invariant sets, showing that synchronized dynamics can occur. Then, stability properties are compared in the cases of quadratic costs and linear costs by considering the degree of product differentiation and the speed of adjustment of prices as key parameters. It is also shown that synchronization takes place if products tend to be relatively complements and stressed similarities and differences between models with quadratic and linear costs. Finally, the paper focuses on the phenomenon of multistability thus underlying new evidences in comparison with the model with linear costs.
\end{abstract}

\footnotetext{
${ }^{a}$ Department of Management, Marche Polytechnic University,

${ }^{b}$ Department of Law, University of Genoa,

${ }^{c}$ Department of Economics and Law, University of Macerata
}

Keywords: Differentiated products; Duopoly; Nonlinear dynamics; Price competition; Quadratic costs; Synchronization; Multistability

JEL Codes: C62; D43; L13

AMS Codes: E32; O41; C62; C63

\footnotetext{
${ }^{*}$ Corresponding author. E-mail: s.brianzoni@univpm.it.
} 


\section{Introduction}

This paper extends the work of Fanti et al. (2013) by considering a nonlinear duopoly with price competition, horizontal differentiation and quadratic production costs (decreasing returns to scale) instead of linear costs (constant returns to scale).

The analysis of economic models with product differentiation has become of greater importance in the oligopoly literature - especially in the case of price-setting firms - both in the absence of managerial delegation contracts (Singh and Vives, 1984) and when ownership and management are separate (Kopel and Lambertini, 2013).

The study of nonlinear dynamics in oligopolies (Bischi et al., 2010) has essentially concentrated on the case of quantity-setting firms to analyse long-term outcomes when information is incomplete (Bischi et al., 1998, 2007), while leaving the case of price-setting firms untreated (an exception is Fanti et al., 2013). The aim of this paper is to deepen the study of the behaviour of nonlinear duopolies with price competition by considering that firms operate with a decreasing returns-toscale technology and incomplete information. The assumption of incomplete information follows the literature pioneered by Bischi et al. (1998). In this case, in fact, firms adopt specific behavioural rules (such as adjustment mechanisms based on marginal profits or the so called Local Monopolistic Approximation, as in Bischi et al., 2007) in the product market.

The present paper stresses the mathematical properties of a duopoly with price competition with nonlinear production costs and then outlines similarities and differences with respect to the model with linear costs. When the cost function is nonlinear we find different dynamic outcomes than when firms operate with a linear cost function. Specifically, we find that synchronized dynamics increases in complexity by starting from the case of independent products to complementary or substitutability. In the case of independent products (i.e., each single firm behaves just like a monopolist in the market), results are different depending on the extent of market demand. In fact, the flip bifurcation for which the Nash equilibrium loses stability occurs earlier when costs are quadratic (resp. linear) if the extent of market demand is large (resp. small).

With regard to multistability, there exist coexisting attractors in either cases of complementarity and substitutability. However, when products are complements the structure of the basins of attraction may result to be complex, so that the final outcome of the economy may be unpredictable. The difference in terms of policy insights is then clear in the two cases. When products are substitutes, it is possible provide adequate policies on the degree of product substitutability (for instance, advertising investments) to drive the economy towards the target, while when products are complements policies may not be effective or have detrimental effects.

The rest of the paper proceeds as follows. Section 2 sets up a duopoly with price competition, linear demand and decreasing returns to scale. It also outlines the two-dimensional map that characterises the evolution of prices from one period to another. Section 3 determines the fixed points and other invariant sets. Then, local stability is investigated and a comparison with the dynamics produced by the model with linear costs is also presented. Similar with the case of linear costs, Section 4 shows that synchronization is likely to emerge if products are complements. In addition, the phenomenon of multistability is investigated, thus underlying new evidences. Specifically, in the particular case of independent products the paper shows that the size of the extent of market demand is crucial to determine whether the primary period doubling bifurcation occurs earlier in the case of quadratic or linear costs. Section 5 outlines the conclusions. 


\section{The model}

The economy is comprised of firms and consumers. There exists a competitive sector that produces the numeraire good $k \geq 0$ (whose price is normalised to 1 ), and a duopolistic sector where firm 1 and firm 2 produce (horizontally) differentiated products of variety 1 and variety 2 , respectively. Let $p_{i} \geq 0$ and $q_{i} \geq 0$ be the price and quantity of product of firm $i(i=1,2)$, respectively.

Consumers. There exists a continuum of identical consumers that have preferences towards goods $q_{1}, q_{2}$ and $k$ described by the separable utility function $V\left(q_{1}, q_{2}, k\right): \mathbb{R}_{+}^{3} \rightarrow \mathbb{R}_{+}$and specified by $V\left(q_{1}, q_{2}, k\right)=U\left(q_{1}, q_{2}\right)+k$, where $U\left(q_{1}, q_{2}\right): \mathbb{R}_{+}^{2} \rightarrow \mathbb{R}_{+}$is a twice differentiable function. The representative consumer aims at maximising $V\left(q_{1}, q_{2}, k\right)$ subject to $p_{1} q_{1}+p_{2} q_{2}+k=M$ (budget constraint), where $M>0$ is the exogenous nominal income of the consumer. This income is high enough to avoid the existence of corner solutions. In addition, there are no income effects on the duopolistic sector. The consumer's optimisation problem is $\max _{\left\{q_{1}, q_{2}\right\}} U\left(q_{1}, q_{2}\right)-p_{1} q_{1}-p_{2} q_{2}+M$. We assume that consumers' preferences towards $q_{1}$ and $q_{2}$ are captured the utility function:

$$
U\left(q_{1}, q_{2}\right)=a\left(q_{1}+q_{2}\right)-\frac{1}{2}\left(q_{1}^{2}+q_{2}^{2}+2 d q_{1} q_{2}\right),
$$

where $a>0$ is the extent of market demand of both goods and $-1<d<1$ is the degree of horizontal product differentiation. If $d=0$ products of variety 1 and variety 2 are independent and each firm behaves as a monopolist. If $d>0$ (resp. $d<0$ ) products are substitutes (resp. complements), while when $d \rightarrow 1$ (resp. $d \rightarrow-1$ ) they tend to be perfect substitutes (resp. perfect complements). By using (1), the consumer's maximisation programme gives the following inverse demands of good 1 and good 2 , respectively:

$$
p_{1}=a-q_{1}-d q_{2} \text { and } p_{2}=a-q_{2}-d q_{1} .
$$

From (2) the corresponding direct demands are then given by:

$$
q_{1}=\frac{a(1-d)-p_{1}+d p_{2}}{1-d^{2}} \text { and } q_{2}=\frac{a(1-d)-p_{2}+d p_{1}}{1-d^{2}} .
$$

Duopolistic firms. We assume that firm $i$ produces with the decreasing returns-to-scale technology $q_{i}=\sqrt{L_{i}}$, where $L_{i}$ is the labour force employed. Firm $i$ 's cost function is $c_{i}=w L_{i}$, where $w>0$ is the cost per unit of labour. The cost function can then be written as $c_{i}=w q_{i}^{2}$, so that average and marginal costs are respectively given by $w q_{i}$ and $2 w q_{i}$, i.e. marginal costs are higher than average costs for every $q_{i}>0$.

Firm $i$ maximises profits $\Pi_{i}=p_{i} q_{i}-w q_{i}^{2}$ with respect to $p_{i}$. Then, by using (3) marginal profits of $i$ th firm are given by

$$
\frac{\partial \Pi_{i}}{\partial p_{i}}=\frac{\left[a(1-d)+d p_{j}\right]\left(1-d^{2}+2 w\right)-2\left(1-d^{2}+w\right) p_{i}}{\left(1-d^{2}\right)^{2}}, \quad i, j=1,2, \quad i \neq j .
$$

Dynamic setting. Consider now a dynamic setting where time is indexed by $t \in \mathbb{Z}_{+}$. By following Bischi et al. (1998) and Fanti et al. (2012, 2013), we assume that both players have limited information. In order to set the price between two subsequent periods, both firms follow an adjustment process based on local estimates of their own marginal profit in the current period. This is given by:

$$
p_{i, t+1}=p_{i, t}+\alpha p_{i, t} \frac{\partial \Pi_{i}\left(p_{i, t}, p_{j, t}\right)}{\partial p_{i, t}}, \quad i=1,2, \quad t \in \mathbb{Z}_{+},
$$


where $\alpha>0$ and $\frac{\partial \Pi_{i}}{\partial p_{i}}$ is determined by (4). The model is definitely described by the following two-dimensional non-invertible discrete time dynamic system $\left(T_{q}, \mathbb{R}_{+}^{2}\right)$ :

$$
T_{q}:\left\{\begin{array}{l}
x^{\prime}=\left[1+\frac{\alpha a}{1+d}+2 \frac{\alpha a w}{(1-d)(1+d)^{2}}\right] x-2\left[\frac{\alpha}{1-d^{2}}+\frac{\alpha w}{\left(1-d^{2}\right)^{2}}\right] x^{2}+\left[\frac{\alpha d}{1-d^{2}}+2 \frac{\alpha d w}{\left(1-d^{2}\right)^{2}}\right] x y \\
y^{\prime}=\left[1+\frac{\alpha a}{1+d}+2 \frac{\alpha a w}{(1-d)(1+d)^{2}}\right] y-2\left[\frac{\alpha}{1-d^{2}}+\frac{\alpha w}{\left(1-d^{2}\right)^{2}}\right] y^{2}+\left[\frac{\alpha d}{1-d^{2}}+2 \frac{\alpha d w}{\left(1-d^{2}\right)^{2}}\right] x y
\end{array},\right.
$$

where $x^{\prime}=p_{1, t+1}, x=p_{1, t}, y^{\prime}=p_{2, t+1}$ and $y=p_{2, t}$.

System $T_{q}$ is a two-dimensional dynamic system whose iteration defines the time evolution of price of each variety in the Bertrand duopoly model with differentiated products and quadratic costs.

In order to analyze dynamics of map $T_{q}$ and compare them with dynamics produced by the model with linear costs, in what follows we refer to the former system with index $q$ and to the latter with index $l$. We underline that the results obtained for the linear case to which we will refer in this paper can be found in Fanti et al. (2013).

\section{Analysis and comparisons}

\subsection{Fixed points and local stability}

A preliminary consideration with regard to system $\left(T_{q}, \mathbb{R}_{+}^{2}\right)$ is that (similar with the model with linear costs) there exist initial conditions $(x(0), y(0)) \in \mathbb{R}_{+}^{2}$ producing trajectories that exit from the set $\mathbb{R}_{+}^{2}$, for instance those belonging to a suitable neighborhood of infinity. This means that a necessary condition for $T_{q}$ to produce meaningful economic dynamics is that the price of each variety at time $t=0$ is not too high.

We recall that a trajectory $\psi_{t}=\{(x(t), y(t))\}_{t=0}^{\infty}$ is said feasible if $(x(t), y(t)) \in \mathbb{R}_{+}^{2}$ for all $t \in N$. Then, we call set $D \subseteq \mathbb{R}_{+}^{2}$, whose points generate feasible trajectories, as the feasible set. With regard to the structure of the feasible set, the following Proposition concerning some limit cases holds.

Proposition 1. (i) If $d \rightarrow 1^{-}$then the set $D-\left\{(0,0),\left(\frac{a w}{1+w}, \frac{a w}{1+w}\right)\right\}$ is empty,

(ii) If $d \rightarrow-1^{+}$then the set $D-\{(0,0),(a, a),(x,-x+2 a)\}$ is empty.

Proof. (i) Consider first $d \rightarrow 1^{-}$, then $\lim _{d \rightarrow 1^{-}} x^{\prime}=-\infty, \forall x(0)>y(0)$ and $\lim _{d \rightarrow 1^{-}} y^{\prime}=-\infty$, $\forall x(0)<y(0)$. Hence initial conditions with $x(0) \neq y(0)$ cannot generate feasible trajectories. Consider now $x(0)=y(0) \neq 0$ and $x(0)=y(0) \neq \frac{a w}{1+w}$. Then $\lim _{d \rightarrow 1^{-}} x^{\prime}=-\infty$. This proves the statement for $d \rightarrow 1^{-}$.

(ii) Case $d \rightarrow-1^{+}$is proved by considering that $\lim _{d \rightarrow-1^{+}} x^{\prime}=\infty \forall x(0) \neq 0, a,-y(0)+2 a$ and $\lim _{d \rightarrow-1^{+}} y^{\prime}=\infty \forall y(0) \neq 0, a,-x(0)+2 a$.

Proposition 1 shows that economic meaningful trajectories are produced only whether the degree of product differentiation is not too high or too low. This confirms the result obtained with linear costs. For intermediate values of $d$, in Figure 1 two different feasible sets are depicted in white, while the set of points generating trajectories that exit from $\mathbb{R}_{+}^{2}$ (i.e. the unfeasible set) is shown in gray. We can observe that if parameter $d$ is slightly modified the structure of the feasible set changes, passing from simple (connected sets) to complex (infinitely many non connected sets). This transition 
(a)

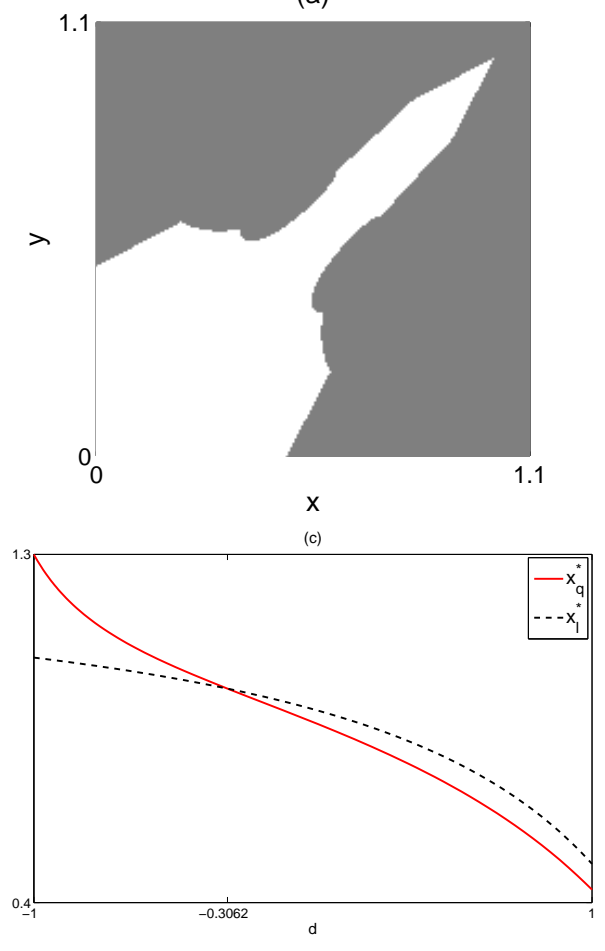

(b)

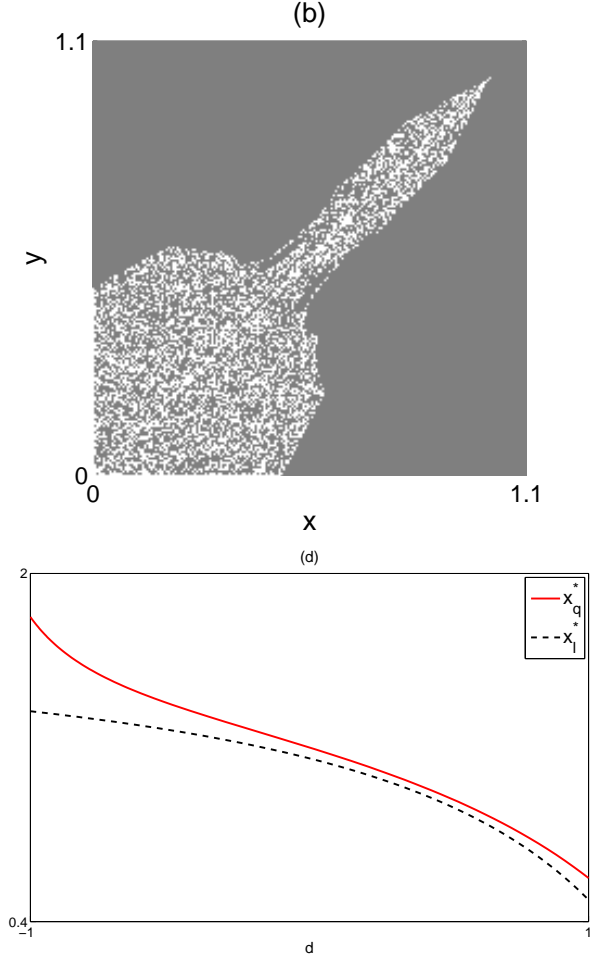

Figure 1: Parameter values: $\alpha=0.5, w=0.5$ and $a=1$. Feasible set (white region) and unfeasible set (gray region) for different values of $d$ point $x_{q}^{*}$ (in red) and $x_{l}^{*}$ (in black) as $d$ is moved, for the parameter values $\alpha=0.5, w=0.5, a=1.3$. $(d)$ The fixed points are computed for $a=1.8$ and the other parameters as in panel $(c)$.

is due to a global bifurcation (namely, a contact bifurcation) that can be investigated by using the method of critical curves, as it has been done in Fanti et al. (2013) (we refer to this work for further details). It is interesting to note that the structure of the feasible set increases in complexity as $d$ increases or decreases and that if products are perfect substitutes or perfect complements almost all trajectories are unfeasible.

Equilibria or fixed points of $T_{q}$ are solutions of the following equation:

$$
T_{q}(x, y)=(x, y)
$$

Trivially, the following proposition holds.

Proposition 2. System $T_{q}$ given by (6) admits four fixed points for all parameter values. They are given by:

$$
\begin{aligned}
& E_{0}=(0,0), \quad E_{1 q}=\left(0, \frac{a(1-d)\left(1-d^{2}+2 w\right)}{2\left(1-d^{2}+w\right)}\right), \quad E_{2 q}=\left(\frac{a(1-d)\left(1-d^{2}+2 w\right)}{2\left(1-d^{2}+w\right)}, 0\right), \\
& \text { and } E_{q}^{*}=\left(x_{q}^{*}, x_{q}^{*}\right)=\left(\frac{a\left(1-d^{2}+2 w\right)}{(1+d)(2-d)+2 w}, \frac{a\left(1-d^{2}+2 w\right)}{(1+d)(2-d)+2 w}\right) .
\end{aligned}
$$

Remark 3. As with linear costs, $T_{q}$ always admits four fixed points: $E_{0}, E_{1 q}$ and $E_{2 q}$ are located on the coordinate axes, $E_{1 q}$ and $E_{2 q}$ are in symmetrical positions with respect to the main diagonal $\Delta=\left\{(x, y) \in \mathbb{R}_{+}^{2}: x=y\right\}$, and $E_{q}^{*} \in \Delta$ is the unique interior Nash equilibrium of the economy. 
By substituting the equilibrium price $x_{q}^{*}$ into direct demand functions we obtain the equilibrium value of the quantity associated with the unique interior fixed point. This is given by

$$
q_{q}^{*}=\frac{a}{(1+d)(2-d)+2 w}>0
$$

Different from the case of linear costs, $q_{q}^{*}$ is positive for all parameter values, while condition $a>w$ must hold to guarantee that the equilibrium quantity when production costs are linear is positive, i.e. $q_{l}^{*}>0$. For this reason we will compare the two models while focusing on the case $a>w$. In addition, we will consider new results for map $T_{q}$ also when $a \leq w$.

In order to compare the position of the interior fixed point on plane $(x, y)$ between the case of linear and quadratic costs, the following Proposition can be proved.

Proposition 4. Let $a>w$. If $a>\frac{17}{8}$ then $x_{q}^{*}>x_{l}^{*}$; if $a \leq 1$ then $x_{q}^{*} \leq x_{l}^{*}$.

Proof. The proof is obtained while comparing $x_{q}^{*}=\frac{a\left(1-d^{2}+2 w\right)}{(1+d)(2-d)+2 w}$ with $x_{l}^{*}=\frac{a(1-d)+w}{2-d}$.

From an economic point of view it is important to stress that the extent of market demand $a$ determines whether prices under decreasing returns to scale are higher or lower than under constant returns to scale. In particular, when market demand is large (resp. small) - i.e. high (resp. low) values of $a$-, equilibrium prices under decreasing returns to scale are higher (resp. lower) than under constant returns to scale. This because when costs are nonlinear and the extent of market demand is large firms operate close to their full production capacity and prices tend to be higher than when costs are linear. However, for intermediate values of $a$, the sign of $x_{q}^{*}-x_{l}^{*}$ is ambiguous as it depends also on the degree of product differentiation $d$. This fact can easily be revealed by comparing Figure $1(c)$ to Figure $1(d)$. In each panel, equilibrium values $x_{q}^{*}$ and $x_{l}^{*}$ are plotted with respect to $d$, while fixing the other parameters and with $a$ being chosen at an intermediate value (i.e. $a \in(1,17 / 8]$ ). It can be observed that if $a=1.3$ (as in panel $(c)$ ) then the equilibrium price in the case of quadratic costs is larger than the equilibrium price in the case of linear costs, as long as the degree of product differentiation $d$ is smaller than the threshold $\tilde{d}=-0.3062$. Differently, if a larger value of $a$ is considered, i.e. $a=1.8$, then $x_{q}^{*}>x_{l}^{*}$ for all $d$-values.

The local stability analysis of the four fixed points of $T_{q}$ can be carried out by considering the Jacobian matrix associated with map $T_{q}$, that is:

$$
\begin{gathered}
J_{q}(x, y)= \\
\left(\begin{array}{ll}
1+\alpha a \frac{\left(1-d^{2}+2 w\right)}{(1-d)(1+d)^{2}}-4 \alpha \frac{\left(1-d^{2}+w\right)}{\left(1-d^{2}\right)^{2}} x+\alpha d \frac{\left(1-d^{2}+2 w\right)}{\left(1-d^{2}\right)^{2}} y & \alpha d \frac{\left(1-d^{2}+2 w\right)}{\left(1-d^{2}\right)^{2}} x \\
\alpha d \frac{\left(1-d^{2}+2 w\right)}{\left(1-d^{2}\right)^{2}} y & 1+\alpha a \frac{\left(1-d^{2}+2 w\right)}{(1-d)(1+d)^{2}}-4 \alpha \frac{\left(1-d^{2}+w\right)}{\left(1-d^{2}\right)^{2}} y+\alpha d \frac{\left(1-d^{2}+2 w\right)}{\left(1-d^{2}\right)^{2}} x
\end{array}\right) .
\end{gathered}
$$

Since the eigenvalues of a diagonal or triangular matrix are given by the elements of the main diagonal, then it can easily be verified that $E_{0}$ is an unstable node while $E_{1 q}$ and $E_{2 q}$ can be both unstable nodes or saddle points. With regard to the local stability properties of the unique interior fixed point $E_{q}^{*}$, the eigenvalues of $J_{q}\left(E_{q}^{*}\right)$ can be obtained. They are given by

$$
\lambda_{\|}\left(E_{q}^{*}\right)=1-\frac{\alpha a\left(1-d^{2}+2 w\right)}{\left(1-d^{2}\right)^{2}} \frac{\left(1-d^{2}\right)(2-d)+2 w}{(1+d)(2-d)+2 w},
$$

and

$$
\lambda_{\perp}\left(E_{q}^{*}\right)=1-\frac{\alpha a\left(1-d^{2}+2 w\right)}{\left(1-d^{2}\right)^{2}} \frac{(1+d)[(1-d)(2+d)+2 w]}{(1+d)(2-d)+2 w} .
$$


Observe that for all parameter values $\lambda_{\|}\left(E_{q}^{*}\right)<1$ and $\lambda_{\perp}\left(E_{q}^{*}\right)<1$, so that the interior fixed point may lose stability if and only if at least one eigenvalue crosses -1 . Furthermore, only the interior fixed point can be attracting, confirming the result in Fanti et al. (2013).

The following Proposition contrasts local stability of the interior fixed point in the cases of quadratic costs $\left(E_{q}^{*}\right)$ and linear costs $\left(E_{l}^{*}\right)$.

Proposition 5. Let $a>w$.

(i) $\forall d \in(-1,1), \exists \bar{\alpha}(d)$ such that if $\alpha>\bar{\alpha}(d)$ then $E_{q}^{*}$ and $E_{l}^{*}$ are both locally unstable;

(ii) $\exists I(0)$ such that $\forall d \in I(0), \exists \tilde{\alpha}(d)$ such that if $\alpha<\tilde{\alpha}(d)$ then $E_{q}^{*}$ and $E_{l}^{*}$ are both locally stable;

(iii) $\exists I(0)$ such that $\forall d \in I(0)$ then $\lambda_{\|}\left(E_{q}^{*}\right)<\lambda_{\|}\left(E_{l}^{*}\right)$ and $\lambda_{\perp}\left(E_{q}^{*}\right)<\lambda_{\perp}\left(E_{l}^{*}\right)$ if and only if a $>\frac{1}{2}$.

Proof. (i) The result is trivially obtained from the following limits: $\lim _{\alpha \rightarrow+\infty} \lambda_{\|}\left(E_{q}^{*}\right)=\lim _{\alpha \rightarrow+\infty} \lambda_{\perp}\left(E_{q}^{*}\right)=$ $-\infty$ and $\lim _{\alpha \rightarrow+\infty} \lambda_{\|}\left(E_{l}^{*}\right)=\lim _{\alpha \rightarrow+\infty} \lambda_{\perp}\left(E_{l}^{*}\right)=-\infty$.

(ii) If $d \rightarrow 0$ we obtain $\lambda_{\|}\left(E_{q}^{*}\right)=\lambda_{\perp}\left(E_{q}^{*}\right)=1-\alpha a(1+2 w):=\lambda\left(E_{q}^{*}\right)$. Then $\lambda\left(E_{q}^{*}\right)>-1$ iff $\alpha<\frac{2}{a(1+2 w)}:=\tilde{\alpha}_{q}$. Similarly, if $d=0, \lambda_{\|}\left(E_{l}^{*}\right)=\lambda_{\perp}\left(E_{l}^{*}\right)=1-\alpha(a+w):=\lambda\left(E_{l}^{*}\right)$ and $\lambda\left(E_{l}^{*}\right)>-1$ iff $\alpha<\frac{2}{a+w}:=\tilde{\alpha}_{l}$. As a consequence, $\tilde{\alpha}(d)=\min \left\{\tilde{\alpha}_{q}, \tilde{\alpha}_{l}\right\}$ which is given by $\tilde{\alpha}_{q}$ (resp. $\tilde{\alpha}_{l}$ ) iff $a \geq \frac{1}{2}$ (resp. $a<\frac{1}{2}$ ).

(iii) The proof immediately follows from part (ii).

Notice that case $(i)$ in Proposition 5 holds also for $a>\bar{a}$ (or $w>\bar{w}$ ), that is $\forall d \in(-1,1) \exists \bar{a}(d)$ (or $\bar{w}(d)$ ) such that $a>\bar{a}$ (or $w>\bar{w}$ ) implies that $E_{q}^{*}$ and $E_{l}^{*}$ are both locally unstable. This means that in both cases of quadratic and linear costs, the Nash equilibrium is unstable when $\alpha$ is large or when the extent of market demand or the cost per unit of labour is high. In fact, if (for instance) the labour cost increases, prices will increase in the next period because they follow the behaviour of marginal cost (i.e., marginal profits becomes large and firms react by setting prices at a level that may cause unstable trajectories in the next period).

Since we are mainly interested in the role played by parameters $d$ and $\alpha$, in Figure $2(a)$ and $(b)$ we depict different bifurcation curves in the parameter plane $(d, \alpha)$ corresponding to set of points such that $\lambda_{\|}\left(E_{q}^{*}\right)=-1$ and $\lambda_{\perp}\left(E_{q}^{*}\right)=-1$ (black curves) or $\lambda_{\|}\left(E_{l}^{*}\right)=-1$ and $\lambda_{\perp}\left(E_{l}^{*}\right)=-1$ (white curves). These curves separate the plane into regions depicted with different colours for which the two fixed points are locally stable, locally unstable or saddle points, respectively. As stated in Proposition 5, we note that both equilibria are locally unstable (resp. stable) if $\alpha$ is large (resp. small) enough, if $d$ belongs to an opportune neighborhood of the origin, while for intermediate values of $\alpha$ several features can be observed depending on the value of the extent of market demand $a$.

\subsection{Invariant sets and synchronized trajectories}

We now consider the invariant sets of system $T_{q}$ and observe that each coordinate semiaxis is invariant, i.e. $T_{q}(x, 0)=\left(x^{\prime}, 0\right)$ and $T_{q}(0, y)=\left(0, y^{\prime}\right)$, as for the linear costs model. As a consequence, also in this case the dynamics of $T_{q}$ on such lines are governed by a one-dimensional map which is topologically conjugate to the standard logistic map. Hence, they can easily be completely known (see Fanti et al., 2013 for details). 

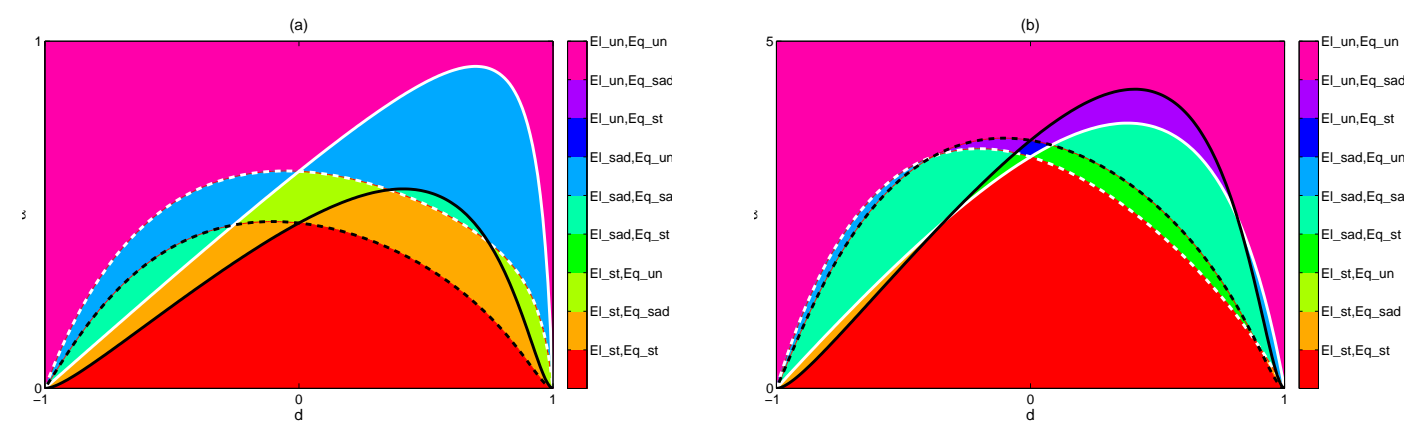

Figure 2: Bifurcation curves on the parameter plane $(d, \alpha)$ identifying regions at which different stability regimes occurs for $w=0.2$. In panel $(a) a=3$ while in panel (b) $a=0.3$. st means locally stable, un means locally unstable while sad means saddle.

Notice that eigenvalues $\lambda_{\perp}$ of $J(0, y)$ and $J(x, 0)$ are given by:

$$
\lambda_{\perp}(x=0)=1+\alpha a \frac{1-d^{2}+2 w}{(1-d)(1+d)^{2}}+\alpha d \frac{1-d^{2}+2 w}{\left(1-d^{2}\right)^{2}} y>1, \quad \forall y \geq 0,
$$

and

$$
\lambda_{\perp}(y=0)=1+\alpha a \frac{1-d^{2}+2 w}{(1-d)(1+d)^{2}}+\alpha d \frac{1-d^{2}+2 w}{\left(1-d^{2}\right)^{2}} x>1, \quad \forall x \geq 0 .
$$

This proves that both invariant semiaxes are repellor by allowing us to restrict attention to the interior fixed point in what follows. In addition, it can be proved that, as for system $T_{l}$, the diagonal $\Delta$ is an invariant set also for $T_{q}$, i.e. $T_{q}(x, x)=\left(x^{\prime}, x^{\prime}\right)$.

In what follows, we focus on the dynamics embedded by $T_{q}$ on $\Delta$ and we compare these ones with those produced by $T_{l}$ along the same restriction. Since $T_{q}(\Delta) \subseteq \Delta$, the dynamics generated by $T_{q}$ on the invariant submanifold $\Delta$ can be studied through the restriction of system $T_{q}$ to set $\Delta$ given by:

$$
T_{q \Delta}=\phi_{q}(x)=\left[1+\frac{\alpha a}{1+d}+\frac{2 \alpha a w}{\left(1-d^{2}\right)(1+d)}\right] x-\alpha \frac{(2-d)(1+d)+2 w}{\left(1-d^{2}\right)(1+d)} x^{2},
$$

that is topologically conjugate to the logistic map $z^{\prime}=\mu_{q} z(1-z)$ with

$$
\mu_{q}=1+\frac{\alpha a}{1-d^{2}}\left(\frac{1-d^{2}+2 w}{1+d}\right)
$$

by the linear transformation

$$
x=\frac{\left(1-d^{2}\right)(1+d)+\alpha a\left(1-d^{2}+2 w\right)}{\alpha((2-d)(1+d)+2 w)} z .
$$

Therefore, the dynamics generated by map $T_{q}$ on the diagonal are completely known, since they can be obtained from those of the logistic map (see Devaney 2003). It is important to observe that equal initial conditions imply equal dynamic behavior forever: trajectories embedded into $\Delta$, i.e. those characterized by $x=y$ for all $t$, are called synchronized trajectories (see Bischi et al. 1998; Bischi and Gardini, 2000).

In order to determine conditions concerning the local stability of the interior fixed point $x_{q}^{*}$ of $\phi_{q}$ and to compare them with the ones holding for the local stability of $x_{l}^{*}$ for $\phi_{l}$, we prove the following Proposition. 
Proposition 6. Let $a>w$.

(a) If $\alpha a(1+2 w)<2$, then $x_{q}^{*}$ is a locally stable fixed point of $\phi_{q} \forall d \in\left(d_{1 q}, d_{2 q}\right),-1<d_{1 q}<0<$ $d_{2 q}<1$.

(b) Let $a \leq \frac{1}{2}$ and $\alpha(a+w)<2$ or $a>\frac{1}{2}$ and $\alpha a(1+2 w)<2$. Then $x_{q}^{*}$ is a locally stable fixed point of $\phi_{q} \forall d \in\left(d_{1 q}, d_{2 q}\right)$ and $x_{l}^{*}$ is a locally stable fixed point of $\phi_{l} \forall d \in\left(d_{1 l}, d_{2 l}\right)$.

Furthermore, let $P=2 a(a-1)(4-\alpha)+\alpha w$ and $Q=4(2 a-1)-a \alpha$. Then:

(i) if $P>0$ and $Q<0$ then $d_{1 q}>d_{1 l}$ and $d_{2 q}>d_{2 l}$;

(ii) if $P<0$ and $Q \neq 0$ then $d_{1 q}<d_{1 l}$ and $d_{2 q}>d_{2 l}$;

(iii) if $P>0$ and $Q>0$ the case is open.

Proof. To prove part $(a)$ assume that $\alpha a(1+2 w)<2$. Then $x_{q}^{*}$ is locally stable iff $\mu_{q} \in(1,3)$, i.e.

$$
f_{q}(d)=\alpha a\left(\frac{1-d^{2}+2 w}{1+d}\right)<2\left(1-d^{2}\right)=g(d) .
$$

Observe that $\lim _{d \rightarrow-1^{+}} f_{q}(d)=+\infty, f_{q}(0)=\alpha a(1+2 w)$ and $f_{q}(d)>0, f_{q}^{\prime}(d)<0$ and $f_{q}^{\prime \prime}(d)>0$ $\forall d \in(-1,1)$. On the other hand $g(d)$ is a concave parabola such that $g(-1)=g(1)=0$ having its maximum at $(0,2)$. Hence, since $\alpha a(1+2 w)<2, f_{q}$ and $g$ intersect each other at two points, namely $d_{1 q}$ and $d_{2 q}$, where $-1<d_{1 q}<0<d_{2 q}<1$.

To prove part $(b)$ we consider the proof of Proposition 3 in Fanti et al. (2013), then conditions $a \leq \frac{1}{2}$ and $\alpha(a+w)<2$ or $a>\frac{1}{2}$ and $\alpha a(1+2 w)<2$ guarantees that the fixed point is locally stable both in the linear and nonlinear costs model, and, in particular, $x_{l}^{*}$ is locally stable $\forall d \in\left(d_{1 l}, d_{2 l}\right)$ where

$$
d_{1 l, 2 l}=\frac{a \alpha \mp \sqrt{a^{2} \alpha^{2}+16-8 \alpha(a+w)}}{4} .
$$

In fact $\mu_{l} \in(1,3)$ iff $f_{l}(d)<g(d)$ where $f_{l}(d)=-a \alpha d+\alpha(a+w)$. Notice also that

$$
f_{q}(d)=f_{l}(d)+h(d), \quad h(d)=\alpha w\left(\frac{2 a}{1+d}-1\right) .
$$

Since $h(d)=0$ iff $d=2 a-1, h^{\prime}(d)<0$ and $h^{\prime \prime}(d)>0 \forall d>-1$, statements $(i)$, (ii) and (iii) can be easily proved.

The previous Proposition states conditions on parameters explaining how the $d$-interval corresponding to the local stability of the unique interior fixed point under linear costs, changes (for instance it enlarges) if quadratic costs are introduced. In Figure $3(d)$ curves $P=0$ and $Q=0$ are depicted on plane $(a, \alpha)$. Observe that if $a$ and $\alpha$ are both small enough, then case $(i i)$ in Proposition 6 holds. Then, the $d$-interval such that the Nash equilibrium attracts synchronized trajectories with quadratic costs is larger with respect to the case of linear costs. The decreasing returns to scale are related to the existence of constraints that prevent some factors of production to increase in their optimal proportions. Thus, given the extent of market demand and the speed of adjustment of price, technological conditions related to quadratic costs allow the stability region (that depends on the degree of product differentiation) to be larger than under constant returns to scale.

In Figure 3, we also show three bifurcation diagrams to compare the long-term evolution of synchronized trajectories in the cases of linear costs (black points) and quadratic costs (red points). 
(a)

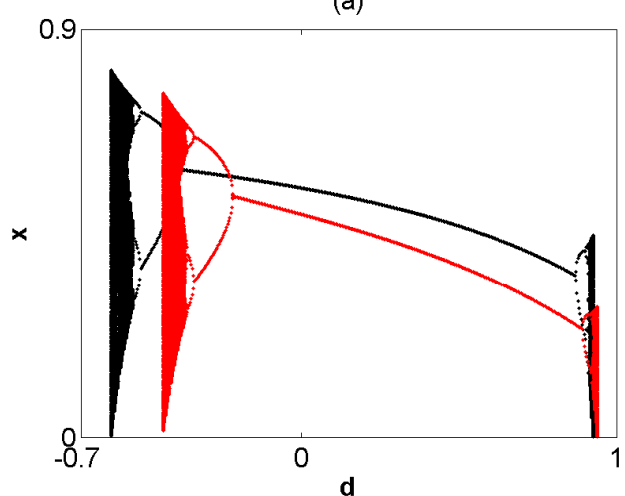

(c)

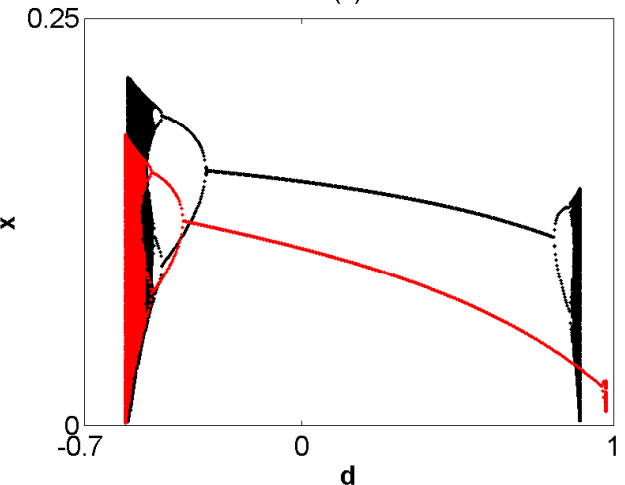

(b)
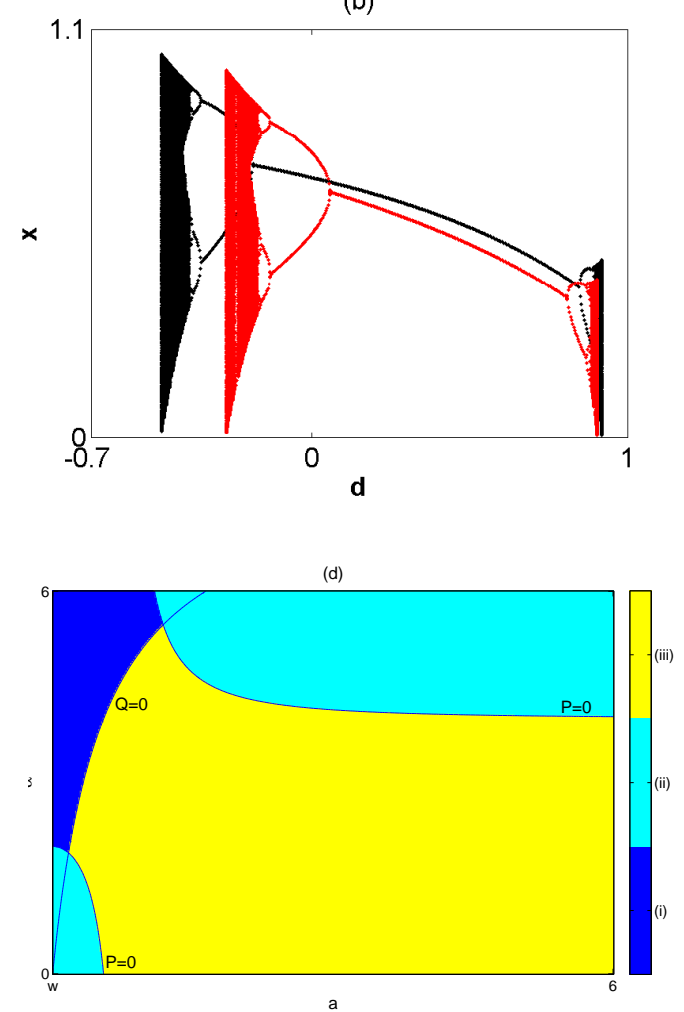

Figure 3: Bifurcation diagrams of map $\phi_{l}$ (in black) and $\phi_{q}$ (in red) w.r.t. $d$ for $\alpha=1.2$ and $w=0.3$ with $x(0)$ the maximun point of $\phi_{l}$ and $\phi_{q}$ respectively. $(a) a=0.8$ and condition (i) of Proposition 6 applies; (b) $a=1.1$ and condition (iii) of Proposition 6 holds. (c) $\alpha=5, a=0.2, w=0.1$ and condition (ii) of Proposition 6 holds. (d) If $w=0.5$ the cases presented in Proposition 6 are considered. 
In panel $(a)$, the $d$-interval such that $x_{q}^{*}$ is locally stable (or, equivalently, $\phi_{q}$ admits an attractor) has moved to the right, confirming the result proved in Proposition $6(i)$. On the other hand, in panel $(b)$ we consider a larger value of $a$ and show a different situation, i.e. the $d$-interval such that $x_{q}^{*}$ is locally stable (or, equivalently, $\phi_{q}$ admits an attractor) is now reduced. Furthermore, if $d=0$ synchronized trajectories converge to a 2-period cycle providing that with quadratic costs fluctuations can be produced also with independent products. This is an important difference with the case of linear costs. In fact, though products are independent when $d=0$ there are endogenous fluctuations even if each firm behaves just like a monopolist in the market (no strategic behaviour). More precisely, the setting of price for the subsequent period by every firm is based on its own marginal profits, so that the effects of nonlinear (quadratic) costs is to induce fluctuations in a parametric context where the fixed point is stable when costs are linear.

Finally, Figure $3(c)$ refers to case $(i i)$ in Proposition 6 and the $d$-interval enlarged when passing from linear to quadratic costs.

We recall that $\forall \mu_{q} \leq 4$ map $T_{q}$ admits an attractor that lies on the diagonal that (given the properties of the logistic map) may consist in either a cycle or a more complex set. By following similar arguments to those used in Proposition 6, and by taking into account numerical evidences shown in Figure 3, the following remark can be stated.

Remark 7. If $a \alpha(1+2 w)<3$ then $\phi_{q}$ admits an attractor $\forall d \in\left(\bar{d}_{1 q}, \bar{d}_{2 q}\right)$. The comparison between the intervals $\left(\bar{d}_{1 q}, \bar{d}_{2 q}\right)$ (which exists in the quadratic costs case) and $\left(\bar{d}_{1 l}, \bar{d}_{2 l}\right)$ (containing the values of $d$ such that an attractor exists in the model linear costs), follows the same properties stated in Proposition 6 .

Finally, about the existence of divergent trajectories, the following Proposition holds.

Proposition 8. A threshold value $\bar{\alpha}_{q}$ does exist such that synchronized trajectories are divergent $\forall \alpha>\bar{\alpha}_{q}$ given the other parameter values (the same result holds if $a>\bar{a}$ or $w>\bar{w}$ ). Let $\bar{\alpha}_{l}$ be the corresponding value of $\bar{\alpha}_{q}$ in the linear costs model. Then: $(i)$ if $w=0$ then $\bar{\alpha}_{q}=\bar{\alpha}_{l}$; (ii) if $w>0$ then $\bar{\alpha}_{q} \geq \bar{\alpha}_{l}$ if and only of $1+d-2 a \geq 0$.

Proof. The synchronized trajectories of $T_{q}$ are governed by $\phi_{q}$ which is topologically conjugate to the logistic map. Hence the generic trajectory diverges if $\mu_{q}>4$, i.e.

$$
\frac{\alpha a}{1-d^{2}} \frac{1-d^{2}+2 w}{1+d}>3
$$

which holds iff

$$
\alpha>3 \frac{\left(1-d^{2}\right)(1+d)}{a\left(1-d^{2}+2 w\right)}:=\bar{\alpha}_{q} .
$$

From Proposition 2 in Fanti et al. 2013 we recall that $\bar{\alpha}_{l}=3 \frac{1-d^{2}}{a(1-d)+w}$ and, after some algebra, parts (i) and (ii) trivially holds.

In line with Bischi et al. (1998), our finding confirms that when at least one of the two players has limited information, the higher the speed of adjustment $\alpha$, the more likely the destabilization of the equilibrium of the map (similar to linear case). Specifically, our results aim at showing that the convergence of synchronized trajectories towards the Nash equilibrium is necessarily associated with intermediate values of $d$, confirming the result obtained with linear costs. In particular, if $x_{q}^{*}$ is locally stable for a given $d$-value, then it loses stability via a period doubling bifurcation due to an increase in the degree of substitutability (resp. complementarity) between products, i.e. $d$ moves to 
1 (resp. to -1). This fact shows that synchronized dynamics increases in complexity while moving from the case of products of independent varieties to complementary or substitutability. In the limit cases $(d \rightarrow \pm 1)$, no bounded dynamics occurs on $\Delta$, confirming the result proved in Proposition 1.

\section{Synchronization and Multistability}

In order to consider producers that start from different initial conditions (i.e., they set different prices at time $t=0)$, we recall that a feasible trajectory starting from $(x(0), y(0)), x(0) \neq y(0)$ is said to synchronize if $|x(t)-y(t)| \rightarrow 0$ as $t \rightarrow+\infty$.

Now, let that the condition stated in Remark 7 hold, so that $\phi_{q}$ admits an attractor $A \subseteq I$, where $I=\left[\phi_{q}^{2}\left(c_{-1}\right), \phi_{q}\left(c_{-1}\right)\right]$ and $c_{-1}$ is the critical point of $\phi_{q}$. Then, $A$ is stable with respect to perturbations along $\Delta$. In order to study the stability of $A$ for $T_{q}$, we have to consider the transverse stability. For fixed points and cycles embedded into the invariant line $\Delta$, the stability conditions along $\Delta$ are the same as for the corresponding fixed points and cycles of the quadratic map $\phi_{q}(x)$. Therefore, in what follows we consider the transverse stability of invariants sets located on $\Delta$.

If the attractor $A$ consists of a fixed point, then by taking into account Proposition 5, if $\alpha$ is not too high and parameter $d$ belongs to a range of intermediate values, then $E_{q}^{*}$ is locally stable and trajectories that start from a neighborhood of $E_{q}^{*}$ synchronizes, i.e. if firms start from different initial conditions, they will behave in the same way in the long term. If $E_{q}^{*}$ loses its local stability, then the following result can be stated.

Proposition 9. (i) Let $a \alpha(1+2 w)<2$. Then $\lambda_{\perp}\left(E_{q}^{*}\right)<\lambda_{\|}\left(E_{q}^{*}\right), \forall d \in I^{+}(0)$ and $\lambda_{\perp}\left(E_{q}^{*}\right)>\lambda_{\|}\left(E_{q}^{*}\right)$, $\forall d \in I^{-}(0)$. (ii) Let $\alpha(a+w)<2$. Then $\lambda_{\perp}\left(E_{l}^{*}\right)<\lambda_{\|}\left(E_{l}^{*}\right), \forall d \in I^{+}(0)$ and $\lambda_{\perp}\left(E_{l}^{*}\right)>\lambda_{\|}\left(E_{l}^{*}\right)$, $\forall d \in I^{-}(0)$.

Proof. (i) Firstly consider the case $d=0$, then $\lambda_{\perp}\left(E_{q}^{*}\right)=\lambda_{\|}\left(E_{q}^{*}\right)=1-a \alpha(1+2 w) \in(-1,1)$ iff $a \alpha(1+2 w)<2$. Hence $E_{q}^{*}$ is locally stable for $d=0$. Consider now $d \neq 0$, hence $\lambda_{\|}\left(E_{q}^{*}\right)-\lambda_{\perp}\left(E_{q}^{*}\right)=$ $F(d) G(d)$ with

$$
F(d):=\frac{a \alpha\left(1-d^{2}+2 w\right)}{\left(1-d^{2}\right)^{2}[(1+d)(2-d)+2 w]} \text { and } G(d):=2 d\left(1-d^{2}+w\right) .
$$

Since $\lim _{d \rightarrow 0} F(d)>0$, then $\exists I(0)$ such that $F(d)>0, \forall d \in I$. Moreover, $G(d)>0$ iff $d>0$, hence the statement is proved. Following similar steps, (ii) is obtained.

Notice that the condition for $E_{q}^{*}$ to be locally stable for $d=0$ is $\alpha a(1+2 w)<2$, i.e. the adjustment coefficient is not too large. According to Proposition 9, if synchronized trajectories converge to $x_{q}^{*}$ with independent products, then trajectories starting from initial conditions close to it, $(x(0), y(0)) \in I\left(E_{q}^{*}, r\right)$, with $x(0) \neq y(0)$, synchronize in the long term as long as $d \in I(0)$ while, as $d$ increases, the fixed point first loses its transverse stability, i.e. trajectories do not synchronize. This fact implies that if there exists a feasible initial condition $(x(0), y(0))$ with $x(0) \neq y(0)$, then it necessarily converges to another bounded attractor $B$ existing out of the diagonal and coexisting with $E_{q}^{*}$. This situation is presented in Figure $4(a)$ : for the chosen parameter constellation, if $d=0$ the Nash equilibrium $E_{q}^{*}$ attracts every initial feasible condition while, if $d$ increases, $E_{q}^{*}$ (the white point) loses its transverse stability and a 2-period cycle is created out of the diagonal (black points); the 2-period cycle attracts all feasible initial conditions having $x(0) \neq y(0)$ (orange region). If $d$ increases further, two cyclic attracting closed invariant curves are created around the unstable 
2-period cycle due to a Neimark-Sacker bifurcation, and non synchronized feasible trajectories (the ones that start from a point in the green region) exhibits a quasi-periodic or periodic behavior (see Figure $4(b)$ ). We also observe that multistability arises, i.e. two attractors coexist, each of which with its own basin of attraction, so that the selected long-term state depends on the initial condition. In this case, the structure of the basins of attraction of different attractors becomes crucial to predict the long-term outcome of the economic system: it may be simple as in Figures $4(a)$ and $(b)$ (hence the final outcome of the economy can be predicted) or more complex, as it will be shown later in this Section.

A different scenario occurs when products are complements, i.e. $d$ decreases. According to Proposition 9 , starting from a situation in which $E_{q}^{*}$ is locally stable for $d=0$ (that is $\alpha$ less enough), when $d$ decreases the Nash equilibrium first loses the stability along the diagonal via a period doubling bifurcation which creates an attracting cycle of period 2. Immediately after this first flip bifurcation, the 2-period cycle is necessarily locally stable (as the transverse eigenvalue is still less then one in modulus). Therefore, synchronization occurs. However, if the degree of horizontal product differentiation still decreases, a sequence of flip bifurcation occurs on the diagonal, and attracting periodic cycles appear around the unstable Nash equilibrium.

If $A$ is an attracting $m$-cycle of $\phi_{q}$, i.e. $A=\left\{x_{1}, \ldots, x_{m}\right\}$, then the corresponding $m$-cycle $\left\{\left(x_{1}, x_{1}\right), \ldots,\left(x_{m}, x_{m}\right)\right\}$ of $T_{q}$ is transversely stable as long as

$$
\lambda_{q \perp}^{(m)}=\prod_{i=1}^{m} \lambda_{q \perp}\left(x_{i}\right) \in(-1,1),
$$

where $\lambda_{q \perp}\left(x_{i}\right)$ are the eigenvalues of the Jacobian matrix evaluated at a point $\left(x_{i}, x_{i}\right)$ associated with eigenvectors normal to $\Delta$. Several numerical experiments show that, similar to what happens for the fixed point, if $A$ is an attracting $m$-cycle for $d=0$, then as $d$ increases (resp. decreases) the $m$-cycle first loses its transverse stability (resp. its stability along $\Delta$ ). As a consequence, we expect synchronization to arise with negative values of $d$, thus confirming the result obtained in Fanti et al. (2013).

If the $m$-cycle is transversely unstable, the situation may become very complicated, as is shown in Figure $4(c)$ where a 4-period cycle attracts all synchronized trajectories, while a 4-piece quasi periodic attractor exists out of the diagonal and synchronization does not take place. A completely different situation is depicted in Figure $4(d)$ : the attractors coexisting on the diagonal and outside the diagonal are complex. In addition, a final bifurcation occurred causing the transition to complex basin boundaries, and consequently to the final state sensitivity, that is the final outcome of the economy becomes unpredictable. Furthermore, it may occur that trajectories starting far from $\Delta$ synchronize in the long term, while firms starting from an initial condition slightly different do not.

Notice that in Figure $4(d) A$ is a 2-piece chaotic attractor on $\Delta$. In order to study its transverse stability, we can apply the procedure proposed in Bischi et al. (1998), Bischi and Gardini (2000) and used also in Fanti et al. (2014). Recall that the transverse Lyapunov exponent is defined as

$$
\Lambda_{q \perp}=\lim _{N \rightarrow \infty} \frac{1}{N} \sum_{n=0}^{N} \ln \left|\lambda_{q \perp}\left(x_{n}\right)\right|,
$$

where $x_{0} \in A$ and $x_{n}$ is a generic trajectory generated by $\phi_{q}$. If $x_{0}$ belongs to a generic aperiodic trajectory embedded inside chaotic set $A$, then $\Lambda_{q \perp}$ is the natural transverse Lyapunov exponent $\Lambda_{q \perp}^{n}$, where natural indicates that the exponent is computed for a typical trajectory taken in the chaotic attractor $A$, where

$$
\Lambda_{q \perp}^{\min } \leq \ldots \leq \Lambda_{q \perp}^{n} \leq \ldots \leq \Lambda_{q \perp}^{\max }
$$


(a)

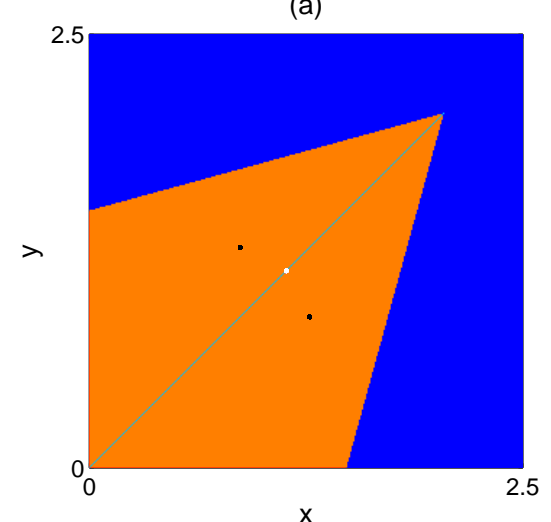

(c)

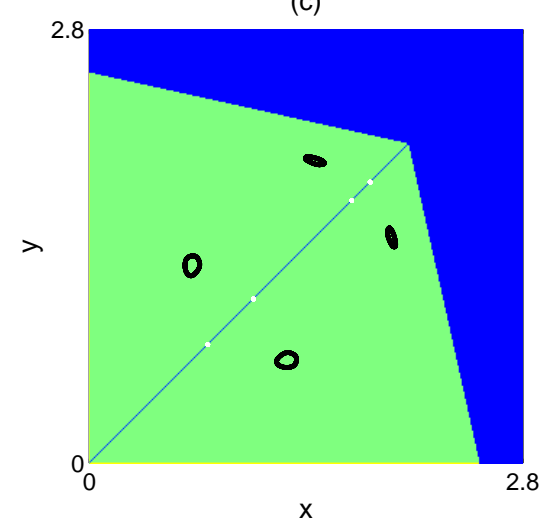

(e)

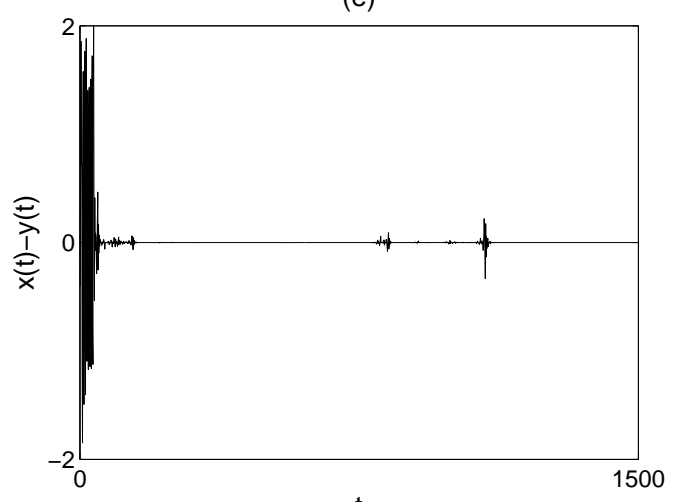

(b)

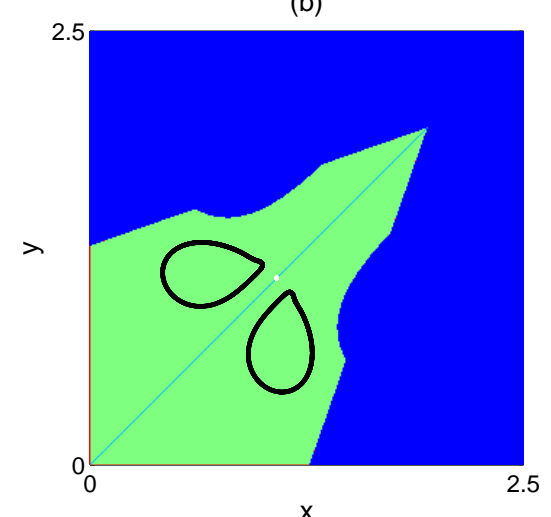

(d)

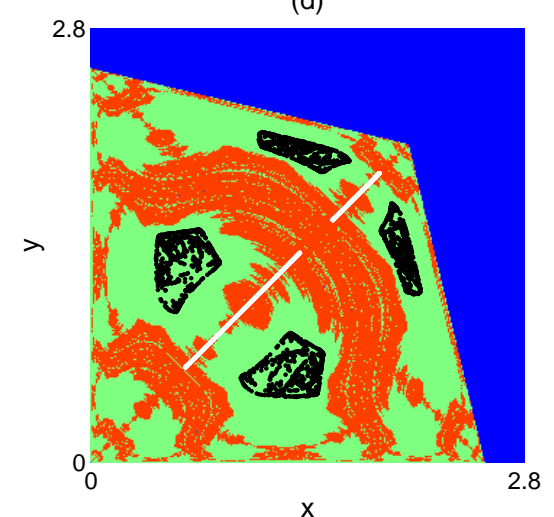

$(\mathrm{f})$

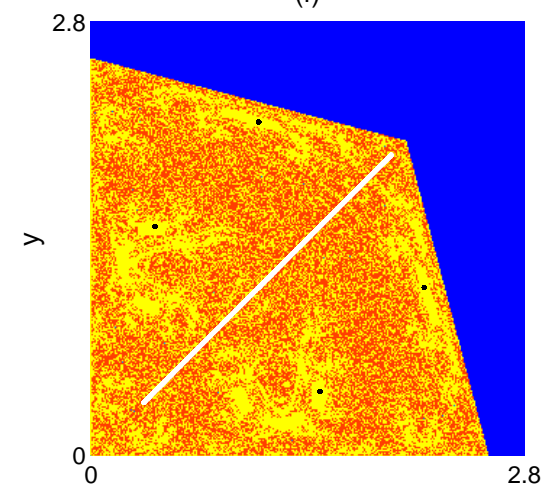

Figure 4: (a) A 2-period cycle (balck points) coexists with the attractor $A \stackrel{\mathrm{x}}{\text { belonging to the diagonal }}$ (the fixed point) for $\alpha=0.4, d=0.4, w=0.5$ and $a=2$. (b) If $d=0.5$ two cyclic attracting closed invariant curves have been created out of the diagonal. (c) If $d=-0.328$ a 4 -piece quasi periodic attractor coexists with a 4-period cycle on the diagonal. $(d)$ If $d=-0.351$, two coexisting complex attractors are owned and the basin structure is quite complex. (e) If $d=-0.383$ bursts away from the diagonal before synchronization occur for $x(0)=1.7$ and $y(0)=2.1$. $(f)$ Complex attractor on $\Delta$ coexisting with a 4 -period cycle for $d=-0.383$. 
is a spectrum of transverse Lyapunov exponents. The natural transverse Lyapunov exponent represents a sort of weighted balance between the transversely repelling and transversely attracting cycles. If all cycles embedded in $A$ are transversely stable, that is $\Lambda_{q \perp}^{\max }<0$, then $A$ is asymptotically stable in the Lyapunov sense. If $\Lambda_{q \perp}^{\max }>0$ and $\Lambda_{q \perp}^{n}<0$ then $A$ is a stable attractor in the Milnor sense. In this last case, transversely repelling trajectories can be re-injected towards $\Delta$ so that their behavior is characterized by some bursts far from the diagonal, before the synchronization or before converging to a different attractor. This situation is called on-off intermittency. This phenomenon is shown in Figure $4(e)$ and a trajectory starting from an initial condition that does not belong to the diagonal has a long transient before converging to $A$. In this case, $A$ is a complex attractor belonging to the diagonal, coexisting with an attracting 4-period cycle whose basin is given by the yellow points in Figure $4(f)$. The economic behaviour cannot be predicted as the basins of attraction have a very complex structure. This result may be of importance for policy insights. For instance, advertising policies may result in undesirable outcomes in the long term, i.e. the convergence towards an attractors where profits are small.

To sum up, it is always possible to have coexisting attractors. If an attractor is complex then initial conditions matter (sensitivity to initial conditions). Indeed, the structure of the attractor is known so we are able to conclude, for instance, that aperiodic fluctuations can occur but they are bounded in a given set. Coexisting attractors can exist in either cases of substitutability and complementarity of products (positive and negative values of $d$, respectively). However, in the case of substitutability it is possible to see - by looking at the basins of attraction - that the final outcome of the economy is predictable. For instance, it is possible to predict long-term dynamics by starting from an initial condition that lies on the diagonal or outside the diagonal. Though coexisting attractors may exist also in the case of complementarity, the structure of the basins of attraction may result to be complex in that case, so that the final outcome the economy may follow is unpredictable (sensitivity with respect to the final outcome), i.e. it is not possible to ascertain whether starting from a given initial condition outside the diagonal the trajectory will lead the economy to an attractor or another (for instance, on the diagonal) that may have a completely different structure, or if the economy will synchronize. The difference in terms of policy insights is then clear in the two cases. When $d>0$ it is possible to drive the direction of long-term outcomes by providing adequate policies on the degree of product substitutability (for instance, advertising investments) as well as on initial conditions, while when $d<0$ policies may not be effective or have detrimental effects.

As is shown above, in a Bertrand duopoly with product differentiation and quadratic costs multistability and synchronization may occur. However, while the synchronization phenomenon has also been described in Fanti et al. (2013), multistability has not been considered in that work, so that we now want to investigate whether this evidence also occurs with linear costs. Obviously, the comparison between the two models becomes quite difficult, as qualitative properties of coexisting attractors as well as properties of their basins are strictly related to the chosen parameter constellations. For this reason, we start the analysis considering parameter sets used in Figure 4 also for the case of linear costs, and then we focus on the particular case of independent products (i.e. $d=0$ ), with the main goal of showing a particular way in which multistability arises and to contrast models with linear and quadratic costs. 
(a)

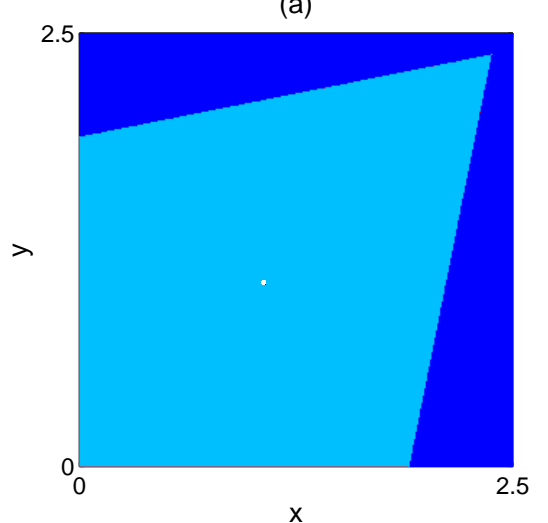

(c)

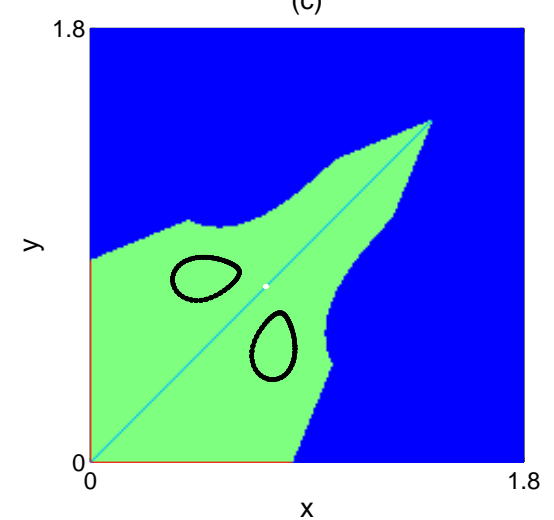

(b)

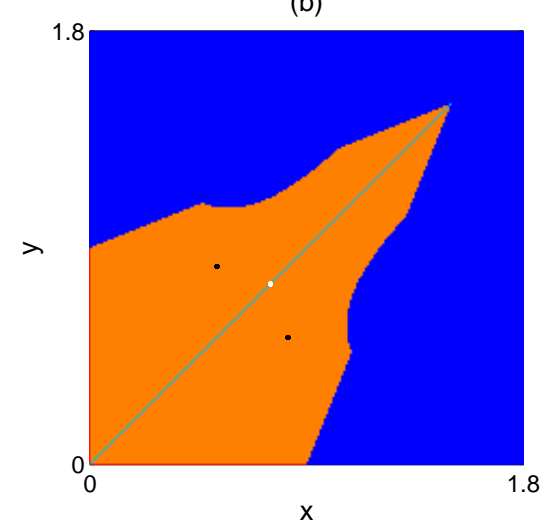

(d)

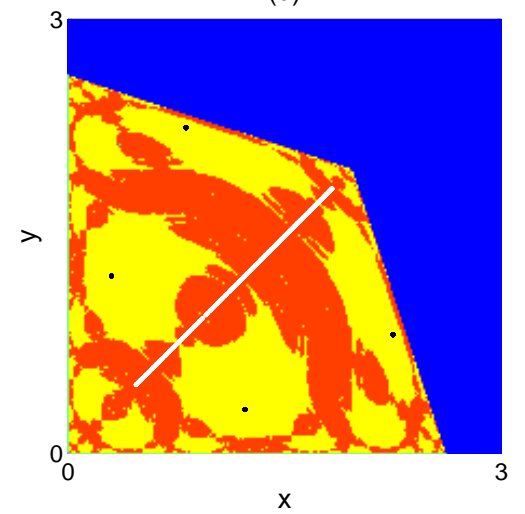

(e)

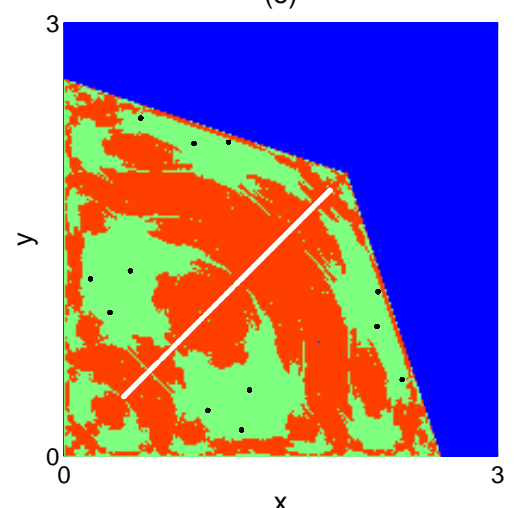

Figure 5: (a) The Nash equilibrium attracts all trajectories starting from and interior feasible point for $\alpha=0.4, d=0.4, w=0.5$ and $a=2$. (b) If $d=0.8$ a 2-period cycle has been created out of the diagonal. (c) If $d=0.82$ two cyclic attracting closed invariant curves have been created out of the diagonal. (c) If $d=-0.662$ a 4 -period cycle coexists with a complex attractor on the diagonal. $(d)$ If $d=-0.667$, a 12-period cycle coexists with a complex attractor on the diagonal. 


\subsection{Multistability with linear costs: numerical comparisons}

Consider first the situation presented in Figure $4(a)$ and observe that $\lambda_{\|}\left(E_{q}^{*}\right) \in(-1,1)$ while $\lambda_{\perp}\left(E_{q}^{*}\right) \notin(-1,1)$. Hence, $E_{q}^{*}$ is a saddle point. By taking into account Proposition 5 part $(i i i)$ and the fact that $\lambda_{\|}\left(E_{l}^{*}\right)<1$ and $\lambda_{\perp}\left(E_{l}^{*}\right)<1$, then $\lambda_{\|}\left(E_{l}^{*}\right) \in(-1,1)$ while $\lambda_{\perp}\left(E_{l}^{*}\right)$ can be both smaller or larger than 1 in modulus. This means that the Nash equilibrium in the case of linear costs may be a saddle point or a stable node. In Figure $5(a)$ it can be observed that all interior feasible points produce trajectories converging to $E_{l}^{*}$, that is a stable node. As for the quadratic costs model, if $d$ increases, $E_{l}^{*}$ looses its transverse stability (see Figure $5(b)$ ). However, this bifurcation occurs later (i.e. at $d \simeq 0.747$ ) providing the following two evidences: with linear costs and substitute products the Nash equilibrium remains stable if $d$ belongs to a $d$-interval which is larger in size with respect to the case of quadratic costs; coexisting attractors emerge while synchronization is avoided. In Figure 5 (c) two cyclical attracting closed invariant curves are presented, similarly to what occurred in the quadratic costs model (see Figure $4(b)$ ).

Consider now the case in which products are complements. As for the quadratic costs model, multistability may emerge and, in addition, synchronization may occur. Anyway, also in this case it can be noticed that, if we consider the same parameter values as in Figure $4(c)$, then the corresponding linear costs model behaves as in Figure $5(a)$, i.e. the Nash equilibrium is a stable node. As a consequence, again we need to further decrease parameter $d$ to obtain situations similar to those presented in $4(c)$ and $(d)$, i.e. showing complex attractors and/or complex basins. In fact, the first period doubling bifurcation creating a 2-period cycle on the main diagonal occurs at $d \simeq-0.5348$, while for smaller values of $d$, the more complex phenomena depicted in Figure $5(d)$ and $(e)$ emerge.

The numerical experiments herewith presented aim at confirming that, for the chosen parameter constellations, multistability occurs and synchronization characterises duopoly models in which products are complements. In addition, the stability of the unique interior Nash equilibrium more likely emerges when production costs are linear.

\subsection{Independent products}

This section studies the particular cases in which firms behave as if they were monopolist and products of each variety are independent, that is $d=0$. In this case our system becomes the following:

$$
T_{q}(d=0):\left\{\begin{array}{l}
x^{\prime}=(1+a \alpha+2 a \alpha w) x-2 \alpha(1+w) x^{2} \\
y^{\prime}=(1+a \alpha+2 a \alpha w) y-2 \alpha(1+w) y^{2}
\end{array},\right.
$$

which is a diagonal system where both equations are conjugated to the logistic map. Since the eigenvalues associated with the Jacobian matrix $J(x, y)$ are $\lambda_{\perp}(x, y)=1+a \alpha(1+2 w)-2 \alpha(1+w) x$ and $\lambda_{\|}(x, y)=1+a \alpha(1+2 w)-2 \alpha(1+w) y$, which are symmetric, any periodic point along the diagonal has always identical eigenvalues. Due to this property, any period doubling bifurcation along the diagonal, which is associated with the bifurcation cascade of the logistic map, is followed by a simultaneous period doubling bifurcation in the symmetric direction. This lead to the particular complex phenomenon of multistability, with the creation of several coexisting attracting cycles that have their own basins of attraction, as in Bischi and Kopel (2003).

Notice that the same holds in the case with linear costs. In particular, the equilibrium $E_{q}^{*}=$ $\left(x_{q}^{*}, x_{q}^{*}\right), x_{q}^{*}=\frac{a(1+2 w)}{2(1+w)}$ loses stability via a first flip bifurcation (with both eigenvalues equal to -1 ) at $\tilde{\alpha}_{q}:=\frac{2}{a(1+2 w)}$; in the same way $E_{l}^{*}$ loses stability at $\tilde{\alpha}_{l}:=\frac{2}{a+w}$. Since we are interested in the 
(a)

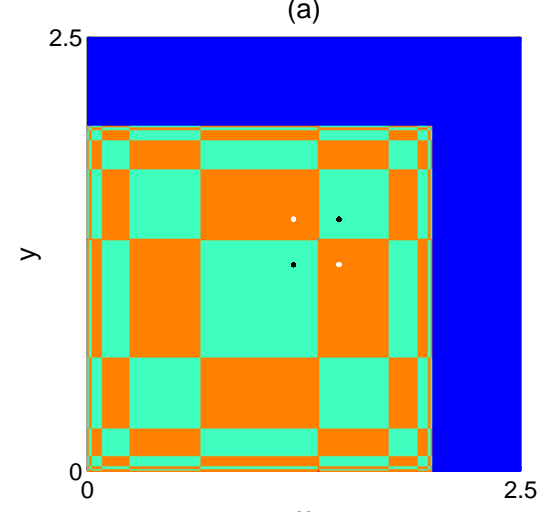

(b)

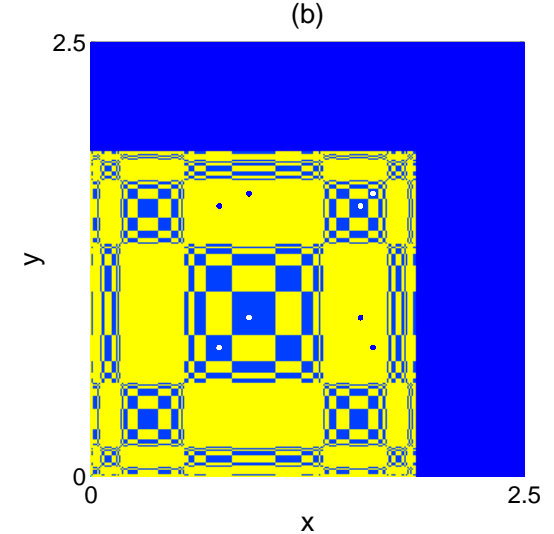

Figure 6: ( $a$ ) If $a=2, w \stackrel{\mathrm{x}}{=} 0.5$ and $\alpha=0.51, T_{q}(d=0)$ admits two stable coexisting cycles of period 2 , whose basins of attraction are represented by the green and orange regions respectively. (b) If $\alpha=0.62, T_{q}(d=0)$ admits two stable coexisting cycles of period 4 , whose basins of attraction are represented by the yellow and blue regions respectively.

comparison between models with linear and quadratic costs, in the following Proposition we consider the first flip bifurcation in both cases.

Proposition 10. Let $d=0, a>w$ and $\tilde{\alpha}_{q}:=\frac{2}{a(1+2 w)}, \tilde{\alpha}_{l}:=\frac{2}{a+w}$. Then:

(i) if $a>\frac{1}{2}$ then $E_{q}^{*}$ and $E_{l}^{*}$ are both locally stable (resp. unstable) $\forall \alpha<\tilde{\alpha}_{q}$ (resp. $\forall \alpha>\tilde{\alpha}_{l}$ ); if $\alpha \in\left(\tilde{\alpha}_{q}, \tilde{\alpha}_{l}\right)$ then $E_{q}^{*}$ is locally unstable while $E_{l}^{*}$ is locally stable;

(ii) if $a<\frac{1}{2}$ then $E_{q}^{*}$ and $E_{l}^{*}$ are both locally stable (resp. unstable) $\forall \alpha<\tilde{\alpha}_{l}$ (resp. $\forall \alpha>\tilde{\alpha}_{q}$ ); if $\alpha \in\left(\tilde{\alpha}_{l}, \tilde{\alpha}_{q}\right)$ then $E_{q}^{*}$ is locally stable while $E_{l}^{*}$ is locally unstable.

Proof. Let $d=0$ then $\lambda_{\perp}\left(E_{q}^{*}\right)=\lambda_{\|}\left(E_{q}^{*}\right)=1-a \alpha(1+2 w)$ which is less then -1 iff $\alpha>\tilde{\alpha}_{q}=\frac{2}{a(1+2 w)}$ while $\lambda_{\perp}\left(E_{l}^{*}\right)=\lambda_{\|}\left(E_{l}^{*}\right)=1-\alpha(a+w)$ which is less then -1 iff $\alpha>\tilde{\alpha}_{l}=\frac{2}{a+w}$. Considering that $\tilde{\alpha}_{q}<\tilde{\alpha}_{l}$ iff $a>\frac{1}{2}$, statements $(i)$ and $(i i)$ are proved.

According to the previous Proposition, the Nash equilibrium loses stability if $\alpha$ increases in both cases of linear and quadratic costs. However, the primary period doubling bifurcation occurs earlier in the case of quadratic (resp. linear) costs if $a>\frac{1}{2}$ (resp. $a<\frac{1}{2}$ ), while it occurs simultaneously in the two cases if $a=\frac{1}{2}$. Interestingly, the extent of market demand differently affects stability of the Nash equilibrium depending on technology conditions. Specifically, if the size of market demand is relatively large and there are decreasing returns to scale (quadratic costs), firms would like to operate at full capacity, i.e. they need small prices and large quantities. However, given the adjustment mechanism based on marginal profits, the higher the extent of market demand, the higher marginal profits and prices in the subsequent period. This over-reaction tend to destabilise the Nash equilibrium. In contrast, when the size of market demand is relatively small and there are constant returns to scale (linear costs), firms would like to operate at average capacity, i.e. they need not too high prices and quantities, otherwise they over-react and fix too high prices in the subsequent period.

Moreover, we note that in the case in which the fixed point is locally unstable, a stable 2-cycle is created along the invariant diagonal $x=y$ and also a stable 2-cycle is created out of the diagonal, with periodic points symmetric to it. In Figure $6(a)$ we consider the case in which the size of demand in the market is great enough; in this case $\tilde{\alpha}_{q}=0.5<\tilde{\alpha}_{l}=0.8$ and if we set $\alpha$ slightly greater then 
$\tilde{\alpha}_{q}$ the first flip bifurcation occurred in the quadratic costs model, while the Nash equilibrium is still stable in the linear costs one.

Similar to what we have done about the first period doubling bifurcation of the fixed point, it is possible to compare the model with quadratic costs with the one with linear costs also in terms of subsequent bifurcations. Consider, for instance, the parameter values for which a second flip bifurcation occurs along the diagonal (which is followed by a simultaneous flip bifurcation out of the diagonal), i.e. $\tilde{\tilde{\alpha}}_{q}:=\frac{\sqrt{6}}{a(1+2 w)}$ for the quadratic costs and $\tilde{\tilde{\alpha}}_{l}:=\frac{\sqrt{6}}{a+w}$ for the linear costs case (these values are obtained by considering $\mu_{q}(d=0)=1+\sqrt{6}$ and $\left.\mu_{l}(d=0)=1+\sqrt{6}\right)$. Again, it is possible to verify that the second flip bifurcation occurs earlier in case of quadratic (resp. linear) costs if $a>\frac{1}{2}$ (resp. $a<\frac{1}{2}$ ). This result holds also for all the subsequent bifurcations. In Figure $6(b)$ we consider a $\alpha$-value slightly greater then $\tilde{\tilde{\alpha}}_{q}$ and, as expected, two stable cycles of period four coexist. Notice that, since $\tilde{\tilde{\alpha}}_{q}=0.6124$, in this situation the Nash equilibrium in the linear costs model is still stable. In fact, as previously discussed, $E_{l}^{*}$ is locally stable as long as $\alpha<0.8$ while, if $\alpha=0.8$, the generic trajectory produced by the model with quadratic costs exit the set $\mathbb{R}_{+}^{2}$. This happens because a final bifurcation occurred for system $T_{q}(d=0)$ when $\alpha=\alpha_{q}^{f}:=\frac{3}{a(1+2 w)}$ (corresponding to $\mu_{q}=4$ ) so that given the chosen parameter constellation, $\alpha_{q}^{f}=0.75$. Notice that when $a>\frac{1}{2}$, the system with quadratic costs closes earlier complexity. This is in line with our previous results and considerations about the effects of the extent of market demand on stability of the Nash equilibrium.

\section{Conclusions}

This paper has taken a dynamic view of a duopoly market with price competition and horizontal differentiation. The literature that studies dynamic phenomena in nonlinear oligopolies has mainly concentrated on the behaviour of quantity-setting firms and provided results about conditions for stability and instability of the Nash equilibrium under complete information (Puu, 1991) and incomplete information by assuming specific behavioural rules (Bischi et al., 1998, 2007; Fanti et al., 2012). The present paper has extended Fanti et al. (2013) and considered firms that operate with a decreasing returns to scale technology that gives rise to nonlinear (quadratic) costs instead of a constant returns to scale technology that gives rise to linear costs. We have remarked differences and similarities of models with quadratic and linear costs, with specific regard to synchronisation and multistability. In particular, when products are substitutes and production costs are linear the Nash equilibrium is stable for a wider range of values of the degree of substitutability than when costs are quadratic. In addition, coexisting attractors emerge while synchronization is avoided. When products are complements the basins of attraction may be complex, so that the existence of different attractors may cause problems of unpredictability.

Finally, when products are independent the flip bifurcation for which the Nash equilibrium loses stability occurs earlier when costs are quadratic (resp. linear) if the extent of market demand is large (resp. small).

Acknowledgements The authors gratefully acknowledge participants at MDEF 2014 held at University of Urbino (Italy). The usual disclaimer applies. 


\section{References}

[1] Bischi, G.I., Chiarella, C., Kopel, M., Szidarovszky, F. (2010): Nonlinear Oligopolies. Stability and Bifurcations. Springer, Berlin, Germany.

[2] Bischi, G.I., Gardini, L. (2000): Global properties of symmetric competition models with riddling and blowout phenomena. Discrete Dynamics in Nature and Society 5, 149-160.

[3] Bischi, G.I., Kopel, M. (2003): Multistability and path dependence in a dynamic brand competition model. Chaos, Solitons and Fractals 18, 561-576.

[4] Bischi, G.I., Naimzada, A.K., Sbragia, L. (2007):Oligopoly games with local monopolistic approximation. Journal of Economic Behavior $\& 3$ Organization 62, 371-388.

[5] Bischi, G.I., Stefanini, L., Gardini, L. (1998): Synchronization, intermittency and critical curves in a duopoly game. Mathematics and Computers in Simulation 44, 559-585.

[6] Devaney, R.L. (2003): An introduction to Chaotic Dynamical Systems, Westview Press, Colorado.

[7] Fanti, L., Gori, L., Sodini, M. (2012): Nonlinear dynamics in a Cournot duopoly with relative profit delegation. Chaos, Solitons and Fractals 45, 1469-1478.

[8] Fanti, L., Gori, L., Mammana, C., Michetti, E. (2013): The dynamics of a Bertrand duopoly with differentiated products: Synchronization, intermittency and global dynamics. Chaos, Solitons and Fractals 52, 73-86.

[9] Fanti, L., Gori, L., Mammana, C., Michetti, E. (2014): Local and global dynamics in a duopoly with price competition and market share delegation. Chaos, Solitons and Fractals 69, 253-270.

[10] Kopel, M., Lambertini, L., (2013): On price competition with market share delegation contracts. Managerial and Decision Economics 34, 40-43.

[11] Puu, T. (1991): Chaos in duopoly pricing. Chaos, Solitons and Fractals 1, 573-581.

[12] Singh, N., Vives, X., 1984. Price and quantity competition in a differentiated duopoly. RAND Journal of Economics 15, 546-554. 